\title{
Coaching educativo en las competencias digitales docentes de la institución educativa San Juan, Trujillo 2021
}

\author{
Segundo Gerardo Ulloa Bocanegra \\ sulloab@ucvvirtual.edu.pe \\ Universidad César Vallejo \\ Dulio Oseda Gago \\ dosedag@ucvvirtual.edu.pe \\ Universidad César Vallejo \\ Trujillo - Perú
}

\section{RESUMEN}

El presente estudio tuvo como objetivo determinar la influencia del coaching educativo en las competencias digitales docentes de los profesores de la I.E. San Juan, Trujillo 2021. La investigación es de tipo básica, con diseño no experimental, transeccional descriptivo correlacional causal. Se aplicó dos cuestionarios, uno de coaching educativo y otro de competencias digitales docentes, a una muestra de 113 docentes. En los resultados se tuvo que en el coaching educativo se muestra un dominio en el nivel alto: 96,5\%, hay un menor dominio en el nivel medio 3,5\% y en las competencias digitales docentes, se muestran un dominio del nivel alto: 77,9\%, hay un menor dominio en el nivel medio 22,1\%. Así mismo, se obtuvo un $r^{2}=0,416$ y el $p<0,01$, el valor del coeficiente rho=0,680., además, el valor de significancia de 0,000 es menor que el nivel de significancia de 0,05. Concluyéndose que el coaching educativo incide en un $41,6 \%$ en las competencias digitales docentes, 58,4\% de las competencias digitales docentes son explicadas por otros factores; existe una relación positiva, alta y estadísticamente muy significativa entre el coaching educativo y las competencias digitales docentes de los profesores en la I.E. San Juan, Trujillo 2021.

Palabras clave: coaching; coaching educativo; competencia; competencia digital 


\title{
Educational coaching in the teaching digital competencies of the educational institution San Juan, Trujillo 2021
}

\begin{abstract}
The present study aimed to determine the influence of educational coaching on the teaching digital competences of the teachers of the I.E. San Juan, Trujillo 2021. The research is of a basic type, with a non-experimental, transectional descriptive correlational causal design. Two questionnaires were applied, one for educational coaching and the other for teaching digital skills, to a sample of 113 teachers. In the results, it was found that educational coaching shows a domain at the high level: $96.5 \%$, there is less domain at the medium level $3.5 \%$ and in teaching digital skills, a domain at the high level : $77.9 \%$, there is less dominance at the middle level $22.1 \%$. Likewise, $\mathrm{r}^{\wedge} 2$ $=0.416$ and $\mathrm{p}<0.01$ were obtained, the value of the coefficient rho $=0.680$. Furthermore, the significance value of 0.000 is less than the significance level of 0.05 . Concluding that educational coaching affects $41.6 \%$ in teaching digital skills, $58.4 \%$ of teaching digital skills are explained by other factors; There is a positive, high and statistically very significant relationship between educational coaching and the teaching digital competencies of teachers in the I.E. San Juan, Trujillo 2021.
\end{abstract}

Keywords: coaching; cducational coaching; competence; digital competence

Artículo recibido: 18. Junio. 2021 Aceptado para publicación: 26. Julio. 2021 Correspondencia: laloulloa@hotmail.com Conflictos de Interés: Ninguna que declarar 


\section{INTRODUCCIÓN}

Actualmente la humanidad atraviesa una pandemia, causada por un letal virus llamado Covid-19 o SARs-Cov-2, el cual ha originado un cambio radical en la existencia de los ciudadanos en todo el orbe, con el propósito de reducir los contagios y disminuir las muertes, producto de este flagelo; las autoridades de la salud de los diversos países recomendaron el aislamiento y distanciamiento social, como medidas que frenen el avance de los contagios. En los sectores educativos, esto propició que se cierren las escuelas, colegios, institutos y universidades, con la consecuente inhabilitación de las clases presenciales, lo cual requirió que las asignaturas migren del modo presencial al remoto (Kikut, 2020).

En muchos países del orbe, las instituciones educativas dieron el giro obligado de las sesiones en forma presencial a una modalidad on-line, lo que se ha realizado de una manera que se podría valorar como admisible genéricamente hablando, es sabido que las acciones a realizar están alineadas a la eventualidad, careciendo de una organización premeditada con antelación para transmitir una materia con métodos online (entornos virtuales). Docentes y estudiantes, por tanto, deben de converger para proporcionar una respuesta donde confluyan decisiones metodológicas y tecnológicas, al mismo tiempo deben garantizar el equilibrio, los derechos y la transparencia para la totalidad de actores internos y externos (García-Peñalvo et al., 2020).

En estos tiempos el término Coaching se ha ocupado de la totalidad de carreras (coaching deportivo, coaching empresarial, etc.). Así surge el Coaching Educativo, donde los profesores mutan su faceta de docente y se vuelcan en ser entrenadores de los estudiantes, buscando potenciar sus habilidades y guiándolos en la consecución y logro óptimo de sus competencias. Este movimiento viene impulsado por Europa, que desea expandirla por los países miembros; ya que han ido estudiando sus efectos en los estudiantes y en su desenvolvimiento, logrando efectos muy agradables. Desde la perspectiva docente, las vitales ventajas del Coaching Educativo son, en primera instancia, un progreso en la correspondencia estudiante-docente, puesto que el profesor abandona su apariencia hostil hacia los educandos para transformarse en el guía que cada estudiante necesita; en segundo término, que el docente obtiene conocimientos novedosos sobre las vivencias reales de sus estudiantes, saliendo a relucir sus desasosiegos y dificultades (Aulasiena, 2020). 
En el Perú, son pequeña minoría, los centros de enseñanza que albergan a profesores que poseen elevadas competencias en las diversas áreas que abarcan su perfil, y en las que se ciñe de forma eficaz y conveniente la utilización y asimilación de las TICS, de acuerdo a la valoración de desempeño ejecutada, Minedu (2017) citado por (Guizado et al., 2019). Los aprendizajes de experiencias pasadas nos han ilustrado que para que los escolares puedan desplegar sus competencias digitales no basta con cambiar la tecnología; hay que cambiar la cultura de las instituciones educativas. En este contexto, los profesores tienen un papel primordial, de ser los primeros en apropiarse de las competitividades necesarias, para poder cumplir a cabalidad con su loable misión formadora (Accinelli, 2019).

En la I.E. Emblemática San Juan de Trujillo, se tiene una serie de inconvenientes relacionados con las competencias digitales docentes, puesto que aún se evidencian deficiencias en un regular número de profesores, con respecto al manejo de las tecnologías y recursos que componen esta competencia, como son: el poder desarrollar efectivamente sesiones de clase remota, saber interactuar reflexivamente en entornos virtuales, poder adaptar con solvencia recursos digitales creados por otros, a las necesidades particulares, respetando los derechos de autor y saber resolver problemas que se puedan presentar con los medios y recursos digitales.

Por todo lo expresado la presente investigación es oportuna pues pretende aportar con la determinación de la influencia del coaching educativo en las competencias digitales de los docentes de la institución educativa emblemática San Juan, de la ciudad de Trujillo, para coadyuvar al cumplimiento de las nuevas metas de la educación básica regular y acorde con las exigencias, producto del estado de emergencia sanitaria.

El problema general de la investigación es el siguiente: ¿existe influencia del coaching educativo en las competencias digitales de los docentes de la institución educativa emblemática San Juan, Trujillo 2021 ?

Esta investigación posee una justificación teórica, pues toda la información empleada para la misma servirá de sustento a futuras investigaciones; una justificación práctica, ya que se determinará la correlación entre el coaching educativo y las competencias digitales docentes, que beneficiarán a los estudiantes; también conserva una justificación metodológica, pues todo el procesamiento y análisis de información, además del diseño y aplicación de instrumentos, se realizará con base al método científico (Sabaj \& Balin, 2012). [trad] 
El objetivo general del estudio es: determinar la influencia del coaching educativo en las competencias digitales de los docentes de la institución educativa emblemática San Juan, Trujillo 2021. La hipótesis general es: el coaching educativo influye significativamente en las competencias digitales de los docentes de la institución educativa emblemática San Juan, Trujillo 2021.

La presente investigación se basó en los siguientes estudios previos internacionales: Hatlevik (2016), en su artículo científico: Examinar la relación entre la autoeficacia de los docentes, su competencia digital, las estrategias para evaluar la información y el uso de las TIC en la escuela. Oslo - Noruega. La investigación concluye que el análisis confirmó que los datos empíricos apoyaban el modelo hipotetizado. Se encontraron cargas factoriales significativas y relaciones positivas entre los factores. En general, los factores del modelo explicaban el $41 \%$ de la competencia digital de los profesores, el $49 \%$ de su autoeficacia en la colaboración en línea y el 36\% de su uso de las TIC en la escuela. [trad]

González (2018), en su artículo científico titulado: Coaching educativo en el mejoramiento del personal docente de la universidad nacional abierta Barinas. Venezuela. El estudio concluye que: El coaching educativo desarrolla el potencial y mejora del rendimiento del educando destacando sus competencias sobre temas como conocimientos, habilidades, destrezas, fomentando un liderazgo a nivel educativo, que aumente su automotivación, su autoestima e implicación en las actividades educativas, en las relaciones personales con sus compañeros y docentes como equipo de trabajo.

A nivel nacional, se tiene a, Osorio (2020), en su tesis titulada: Coaching educativo y competencias digitales en docentes de la Institución Educativa Emblemática Pedro Adolfo Labarthe, La Victoria- 2019. Lima. La investigación concluye que el coaching educativo tiene una baja correlación con las competencias digitales en docentes de la I.E.E. Pedro Adolfo Labarthe. El coeficiente Rho de Spearman de 0.213 implica una baja correlación, además siendo el nivel de significancia bilateral $(\mathrm{Sig}=\mathrm{p}=0.044<0.05$. rechazando H0). Por ende: existe relación significativa entre coaching educativo y las competencias digitales en pedagogos de la mencionada Institución Educativa.

A su vez, Farroñay (2019) en su investigación titulada: El impacto del coaching en el desarrollo de las competencias básicas en los docentes de la Institución Educativa Maynas, Iquitos 2017, la cual tuvo como objetivo determinar la relación entre el coaching 
y las competencias básicas en los docentes. Los resultados arrojaron a través del Coeficiente de Correlación de Spearman un valor $r=0,724$, lo que significa que existe una relación significativa del impacto del coaching en el desarrollo de las competencias básicas en los docentes. En tanto, la importancia de la formación docente y los aprendizajes a lo largo de toda la vida es uno de los tópicos aireados en los organismos educativos internacionales.

Respecto a las bases teórico científicas, para Teeman et al. (2011) citados por (Sánchez \& Boronat, 2014), el coaching educativo es una herramienta para la formación, que emerge como un tipo de estrategia y sistema, encontrándose dentro del entorno pedagógico; encaminada a los docentes, basado en la academia, colaborativa y conducente al aprendizaje de los estudiantes.

En cuanto a las competencias digitales del docente, Ala-Mutka et al. (2008) citados por (Martínez-Garcés \& Garcés-Fuenmayor, 2020, pág. 3), las definen “como su habilidad para la comprensión, uso y evaluación crítica de medios digitales de comunicación”.

Por consiguiente, podemos afirmar que el tema no es reciente, sin embargo, todavía hay mucho trabajo que realizar en lo concerniente a la formación de profesores con el dominio de las competencias digitales que el presente siglo requiere con suma urgencia, al respecto, Fernández-Cruz \& Fernández-Díaz (2016, pág. 104) nos manifiestan la siguiente apreciación "Claramente, los profesores no pueden ayudar a los estudiantes a desarrollar una competencia que ellos mismos no poseen en profundidad". [trad]

\section{ESTRATEGIAS METODOLÓGICAS}

La investigación realizada es básica, determinada a un juicio más acabado por medio del entendimiento de matices esenciales de los fenómenos, de las situaciones evidentes o de las correspondencias establecidas por los elementos (Consejo Nacional de Ciencia, Tecnología e Innovación Tecnológica, 2018). Según Hernández et al. (2014), el diseño del estudio es transeccional correlacional causal, pues refieren correspondencias entre dos o más variables en un tiempo preciso, ya sea en forma correlacional o acorde a la correspondencia motivo -consecuencia.

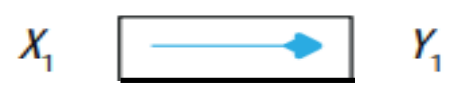

Figura 1: Diseño de investigación, fuente Metodología de la investigación, (Hernández et al., 2014) 
Donde:

$$
\begin{aligned}
\mathrm{X} 1 & =\text { coaching educativo. } \\
\mathrm{Y} 1 & =\text { competencias digitales docentes. } \\
\longrightarrow & =\text { Causalidad. }
\end{aligned}
$$

La población objeto de estudio del presente trabajo estuvo comprendida por 127 docentes y la muestra por 113 docentes. El muestreo fue probabilístico, con un nivel de significancia del $2,5 \%$.

\section{RESULTADOS Y DISCUSIÓN}

A continuación, se presentan los resultados de las variables: coaching educativo y competencias digitales docentes, aplicados a los 113 docentes de la institución educativa emblemática San Juan, Trujillo 2021; los cuales se visualizan en las siguientes tablas.

Tabla 1: Niveles de la variable independiente: Coaching educativo

\begin{tabular}{|l|c|c|}
\hline \multicolumn{1}{|c|}{ Niveles } & Frecuencia & Porcentaje \\
\hline Bajo & 0 & 0,00 \\
\hline Medio & 4 & 3,50 \\
\hline Alto & 109 & 96,50 \\
\hline Total & 113 & 100,00 \\
\hline
\end{tabular}

Nota: Data de coaching educativo.

De la tabla 1, se puede deducir que después de haber aplicado el instrumento de la variable coaching educativo a los 113 docentes de la I.E.E. San Juan, Trujillo 2021, se tiene que 109 docentes que representa el 96,50\% estuvieron en el nivel alto, luego 4 docentes que es el 3,50\% en el nivel medio, no hubo docentes en el nivel bajo. Al respecto de estos resultados para Teeman et al. (2011) citados por (Sánchez \& Boronat, 2014), el coaching educativo es una herramienta para la formación, que emerge como un tipo de estrategia y sistema, encontrándose dentro del entorno pedagógico; encaminada a los docentes, basado en la academia, colaborativa y conducente al aprendizaje de los estudiantes.

Tabla 2: Niveles de la variable dependiente: Competencias digitales docentes

\begin{tabular}{|l|c|c|}
\hline \multicolumn{1}{|c|}{ Niveles } & Frecuencia & Porcentaje \\
\hline Bajo & 0 & 0,00 \\
\hline Medio & 25 & 22,10 \\
\hline Alto & 88 & 77,90 \\
\hline Total & 113 & 100,00 \\
\hline
\end{tabular}

Nota: Data de competencias digitales docentes 
De la tabla 2, se deduce que después de haber aplicado el instrumento de la variable competencias digitales docentes, específicamente en la muestra de 113 docentes de la institución educativa emblemática San Juan, Trujillo 2021, se tiene que 88 docentes que representa el 77,90\% estuvieron en el nivel alto, luego 25 docentes que es el 22,10\% en el nivel medio, en el nivel bajo no se tuvo a ningún docente. Cabe hacer referencia a Fernández-Cruz y Fernández-Díaz (2016, pág. 104), quienes nos hacen reflexionar "Claramente, los profesores no pueden ayudar a los estudiantes a desarrollar una competencia que ellos mismos no poseen en profundidad". [trad]

Ahora pasaremos a realizar la prueba de hipótesis:

Hipótesis nula $\left(\boldsymbol{H}_{\mathbf{0}}\right)$ : el coaching educativo no influye significativamente en las competencias digitales de los docentes de la I.E. San Juan, Trujillo 2021.

Hipótesis alterna $\left(\boldsymbol{H}_{1}\right)$ : el coaching educativo influye significativamente en las competencias digitales de los docentes de la I.E. San Juan, Trujillo 2021.

Nivel de significancia o riesgo: $\alpha=0,01=1 \%$.

Tabla 3: Correlación de Coaching Educativo y las Competencias Digitales Docentes

\begin{tabular}{||l|l|c|}
\hline \multirow{2}{*}{ CE } & Coeficiente de correlación & CDD \\
\cline { 2 - 3 } & Sig. (bilateral) &, $680^{* *}$ \\
\cline { 2 - 3 } & $\mathrm{N}$ &, 000 \\
\hline
\end{tabular}

Nota: Data de Coaching Educativo y Competencias Digitales Docentes

Tabla 4: Efecto del Coaching Educativo en las Competencias Digitales Docentes

\begin{tabular}{|c|c|c|c|c|}
\hline Modelo & $\mathbf{R}$ & R cuadrado & R cuadrado corregida & $\begin{array}{c}\text { Error típ. de la } \\
\text { estimación }\end{array}$ \\
\hline 1 &, $645 \mathrm{a}$ &, 416 &, 411 & 9,799 \\
\hline
\end{tabular}

Nota: Data de Coaching Educativo y Competencias Digitales Docentes

Decisión estadística: Dado que (p-valor: 0,000<0,010) y un $r^{2}=0,416$, podemos afirmar que se rechaza la hipótesis nula $\left(H_{0}\right)$ y se acepta la hipótesis alterna $\left(H_{1}\right)$.

Conclusión estadística: Se concluye que existe efecto positivo $(41,6 \%)$ y altamente significativo del coaching educativo en las competencias digitales de los docentes de la I.E. San Juan, Trujillo 2021. 
Luego de haberse realizado el procesamiento de datos en las dos variables de la investigación, corresponde la realización de la discusión de resultados. Estadísticamente se tiene que (p-valor: $0,000<0,010$ ), en consecuencia, se rechaza la hipótesis nula (Ho) y se acepta la hipótesis alterna (Hi), se concluye que el coaching educativo influye significativamente en las competencias digitales de los docentes de la I.E. San Juan, Trujillo 2021. Se interpreta de acuerdo a los resultados, que el coaching educativo incide en un $41,6 \%$ en las competencias digitales docentes, $58,4 \%$ de las competencias digitales docentes son explicadas por otros factores. Además, existe una alta relación directa, muy significativa entre el coaching educativo y las competencias digitales docentes de los profesores de la I.E. estudiada.

Estos resultados son similares con los hallados por: Farroñay (2019), en su tesis doctoral: El impacto del coaching en el desarrollo de las competencias básicas en los docentes de la Institución Educativa Maynas, Iquitos 2017; concluye que el impacto del coaching incide en un 52,4\% en las competencias básicas de los docentes, existe una relación significativa del impacto del coaching en el desarrollo de las competencias básicas en los docentes., con $\mathrm{r}^{2}=0,524$, un Rho de Spearman $=0,724$.

Se asemejan también con lo hallado por (Hatlevik, 2016), en su artículo científico: Examinar la relación entre la autoeficacia de los docentes, su competencia digital, las estrategias para evaluar la información y el uso de las TIC en la escuela; quien concluyó que en general, los factores del modelo explicaban el $41 \%$ de la competencia digital de los profesores, el $59 \%$ eran explicadas por otros factores.

Son mejores que los hallados por Osorio(2020), en su investigación titulada: Coaching educativo y competencias digitales en docentes de la Institución Educativa Emblemática Pedro Adolfo Labarthe, La Victoria- 2019, donde concluye, que el coaching educativo tiene una moderada correlación con las competencias digitales docentes, dado por un $\mathrm{r}=$ 0,486 , lo que hace suponer, un $\mathrm{r}^{2}=0,2361$, que indicaría que el coaching académico influye en un $23,6 \%$ en las competencias digitales docentes y el $76.4 \%$ de estas, son explicadas por otros factores. La explicación de la mejoría de los resultados de la presente investigación con respecto a la Osorio, se debe al espacio temporal de la realización de las mismas, puesto que como es sabido esta emergencia sanitaria producto de la pandemia obligó a dar un giro en las labores educativas, teniendo los docentes la imperiosa 
necesidad de adquirir las competencias digitales necesarias para poder desarrollar su labor con eficiencia.

Por tanto, afirmamos que hay influencia y relación entre las variables (en mayor o menor grado), cumpliéndose lo estipulado teóricamente, así según los aportes de Desimone y Pak (2016), quienes sostienen, que el coaching educativo, es un afán interdisciplinario que se ha afirmado en los colegios como un medio para el estímulo de nuevos docentes, el aprendizaje continuo de los mismos, la ayuda en la ejecución de novedosas decisiones y últimamente para ayudar a los profesores a intuir y adecuar su ejercicio a la normatividad estatal. En cuanto a las competencias digitales del docente, Ala-Mutka et al. (2008) citados por (Martínez-Garcés \& Garcés-Fuenmayor, 2020, pág. 3), lo definen “como su habilidad para la comprensión, uso y evaluación crítica de medios digitales de comunicación". Es allí donde incide el coaching educativo y sus dimensiones, en las habilidades de los docentes, orientándolas al uso reflexivo de las tecnologías digitales, consolidando la competencia digital de los mismos.

Por último, los resultados se armonizan con lo hallado por González (2018), en su artículo científico: Coaching educativo en el mejoramiento del personal docente de la universidad nacional abierta Barinas; concluyó que el coaching educativo desarrolla el potencial y mejora del rendimiento del educando destacando sus competencias sobre temas como conocimientos, habilidades, destrezas, fomentando un liderazgo a nivel educativo, que aumente su automotivación, su autoestima e implicación en las actividades educativas, en las relaciones personales con sus compañeros y docentes como equipo de trabajo. Por lo tanto, opino al respecto que el coaching tiene la capacidad de afinar la productividad de las personas en diversos campos como los de la inteligencia, destrezas, habilidades; además que fomenta el liderazgo y fortalece las relaciones interpersonales con los colegas y maestros.

Finalmente sostenemos que es muy recomendable aplicar estrategias de coaching educativo en las instituciones académicas, puesto que como se ha demostrado hay influencia de esta variable, sobre las competencias digitales de los maestros; y sería un gran avance para garantizar que los estudiantes reciban "instrucción de calidad" de parte de sus docentes, puesto que estos han adquirido y potenciado las habilidades tan requeridas en este presente siglo XXI. 


\section{CONCLUSIONES}

- Se ha hallado con un nivel de significancia del $1 \%$ que el coaching educativo incide en un $41,6 \%$ en las competencias digitales docentes, $58,4 \%$ de las competencias digitales docentes son explicadas por otros factores.

- La conciencia incide en un $34,6 \%$ en las competencias digitales docentes de los profesores de la I.E. San Juan, Trujillo 2021.

- La autocreencia incide en un $31,1 \%$ en las competencias digitales docentes de los profesores de la I.E. San Juan, Trujillo 2021.

- La responsabilidad incide en un 36,9\% en las competencias digitales docentes de los profesores de la I.E. San Juan, Trujillo 2021.

\section{LISTA DE REFERENCIAS}

Accinelli, A. (2019). Educación para un mundo digital. Recuperado el 11 de Diciembre de 2020, de https://www.gob.pe/institucion/minedu/informespublicaciones/345597-educacion-para-un-mundo-digital

Aulasiena. (2020). 4 Beneficios del coaching educativo que como docente deberías conocer. Recuperado el 10 de Diciembre de 2020, de https://aulasiena.com/httpaulasiena-com-experto-coaching-educativo/

Consejo Nacional de Ciencia, Tecnología e Innovación Tecnológica. (2018). Portal CONCYTEC. Obtenido de https://portal.concytec.gob.pe/index.php/informacioncti/reglamento-del-investigador-renacyt

Desimone, L., \& Pak, K. (2016). Instructional Coaching as High-Quality Professional Development. Theory Into Practice, 1-10. doi:http://dx.doi.org/10.1080/00405841.2016.1241947

Farroñay, K. (2019). El impacto del coaching en el desarrollo de las competencias básicas en los docentes de la Institución Educativa Maynas. Iquitos - 2017. Lima: UNIVERSIDAD NACIONAL DE EDUCACIÓN - Enrique Guzmán y Valle.

Fernández-Cruz, F., \& Fernández-Díaz, M. (2016). Generation Z's Teachers and their Digital Skills. Comunicar, 24(46), 97-105. doi:10.3916/C46-2016-10

García-Peñalvo, F., Corell, A., Abella-García, V., \& Grande, M. (2020). La evaluación online en la educación superior en tiempos de la COVID-19. Education in the Knowledge Society, 21(12), 1-26. doi:10.14201/eks.23013 
González, R. (2018). Coaching educativo en el mejoramiento del personal docente de la universidad nacional abierta Barinas. Remembranza, 1(1), 114-123.

.Guizado, F., Menacho, I., \& Salvatierra, A. (2019). Competencia digital y desarrollo profesional de los docentes de dos instituciones de educación básica regular del distrito de Los Olivos, Lima-Perú. Hamut'ay, 6(1), 54-70. doi:http://dx.doi.org/10.21503/hamu.v6i1.1574

Hatlevik, O. (2016). Examining the Relationship between Teachers' Self-Efficacy, their Digital Competence, Strategies to Evaluate Information, and use of ICT at School. Scandinavian Journal of Educational Research, 1-13. doi:10.1080/00313831.2016.1172501

Hernández, R., Fernández, C., \& Baptista, M. (2014). METODOLOGíA DE LA INVESTIGACIÓN (Sexta ed.). México: McGRAW-HILL/ INTERAMERICANA EDITORES, S.A. DE C.V.

Kikut, L. (2020). Análisis de resultados de la evaluación de la virtualización de cursos en la UCR ante la pandemia por COVID-19: Perspectiva estudiantil. Centro de evaluación académica. San José: Universidad de Costa Rica. Obtenido de http://hdl.handle.net/10669/81216

Martínez-Garcés, J., \& Garcés-Fuenmayor, J. (2020). Competencias digitales docentes y el reto de la educación virtual derivado de la covid-19. Educación y Humanismo, 22(39), 1-16. doi:10.17081/eduhum.22.39.4114

Osorio, Z. (2020). Coaching educativo y competencias digitales en docentes de la Institución Educativa Emblemática Pedro Adolfo Labarthe, La Victoria- 2019. Lima, Perú: UCV.

Sabaj, O., \& Balin, D. (2012). Description of the Justification Forms in Research Articles in Spanish in Six Scientific Areas. Onomazein, 25(1), 315-344.

Sánchez, B., \& Boronat, J. (2014). Coaching Educativo: Modelo para el desarrollo de competencias intra e interpersonales. Educación XX1, 17(1), 221-242. doi:10.5944/educxx1.17.1.1072 